\title{
GENERALIZATION OF FUZZY OPERATIONS USING ARBITRARY COMPLEMENT FUNCTION
}

\author{
P. Xavier ${ }^{1}$, P. Thangavelu ${ }^{2}$ \\ ${ }^{1}$ Department of Mathematics \\ Karunya University \\ Coimbatore, 641114, Tamilandu, INDIA \\ ${ }^{2}$ Ramanujam Centre for Mathematical Sciences \\ Thirupuvanam, 630611, Tamilnadu, INDIA
}

\begin{abstract}
The notion of fuzzy $\mathscr{C}$-interior and fuzzy $\mathscr{C}$-exterior are introduced using the arbitrary complement function $\mathscr{C}:[0,1] \rightarrow[0,1]$ and their properties are studied. Further new fuzzy topologies are characterized using the fuzzy $\mathscr{C}$-closure operator, fuzzy $\mathscr{C}$-interior operator and fuzzy $\mathscr{C}$-exterior operator.
\end{abstract}

AMS Subject Classification: 54A40, 03E72

Key Words: fuzzy sets, fuzzy complement functions, fuzzy $\mathscr{C}$-interior, fuzzy $\mathscr{C}$-exterior, fuzzy topology

\section{Introduction}

Chang [2] introduced fuzzy topological space by generalizing the concepts of point set topology. In Chang's topology the relationship between the fuzzy open sets and fuzzy closed sets are defined using the standard complement $C(x)=1-x, 0 \leq x \leq 1$. Bageerathi and Thangavelu [8] generalized the fuzzy closed sets into fuzzy $\mathscr{C}$-closed sets using the arbitrary complement function $\mathscr{C}:[0,1] \rightarrow[0,1]$. A fuzzy subset $\lambda$ of a fuzzy topological space $X$ is fuzzy $\mathscr{C}$-closed if $\mathscr{C} \lambda$ is fuzzy open in $X$. Xavier and Thangavelu[12] defined fuzzy $\mathscr{C}$ -

Received: $\quad$ September 15, 2016

Revised: $\quad$ November 23, 2016

Published: $\quad$ February 1, 2017

$\S_{\text {Correspondence author }}$ (c) 2017 Academic Publications, Ltd. url: www.acadpubl.eu 
open sets using the arbitrary complement function $\mathscr{C}$ which characterizes fuzzy topological space, fuzzy continuity and fuzzy compactness. The computation of fuzzy interior, closure, boundary and exterior provide many solutions to problems in image processing [3],[10] and spatial objects in GIS [6],[7][11]. In this paper we define the fuzzy $\mathscr{C}$-interior, fuzzy $\mathscr{C}$-exterior and their properties are investigated. It leads to the characterization of new fuzzy topologies using fuzzy $\mathscr{C}$-interior operator, fuzzy $\mathscr{C}$-exterior operator and $\mathscr{C}$-closure operator.

\section{Preliminaries}

This section contains some basic notions and results that are used in this paper. The definitions of fuzzy sets and fuzzy topological spaces are found in [1], [2]. Fuzzy subsets in $X$ will be denoted by $\lambda$ and $\mu$.

Definition 1. [5] Let $\mathscr{C}:[0,1] \rightarrow[0,1]$ be a complement function. If $\lambda$ is a fuzzy subset of $(X, \tau)$ then the complement $\mathscr{C} \lambda$ of a fuzzy subset $\lambda$ is defined by $\mathscr{C} \lambda(x)=\mathscr{C}(\lambda(x))$ for all $x \in X$. A complement function $\mathscr{C}$ is said to satisfy

(i) the boundary condition if $\mathscr{C}(0)=1$ and $\mathscr{C}(1)=0$,

(ii) monotonic condition if $x \leq y \Rightarrow \mathscr{C}(x) \geq \mathscr{C}(y)$, for all $x, y \in[0,1]$,

(iii) involutive condition if $\mathscr{C}(\mathscr{C}(x))=x$, for all $x \in[0,1]$.

Lemma 2. [8] Let $\mathscr{C}:[0,1] \rightarrow[0,1]$ be a complement function that satisfies involutive and monotonic conditions. Then for any family $\left\{\lambda_{\alpha}: \alpha \in \Delta\right\}$ of fuzzy subsets of $\mathrm{X}$, we have

$$
\mathscr{C}\left(\vee\left\{\lambda_{\alpha}: \alpha \in \Delta\right\}\right)=\wedge\left\{\mathscr{C} \lambda_{\alpha}: \alpha \in \Delta\right\}
$$

and

$$
\mathscr{C}\left(\wedge\left\{\lambda_{\alpha}: \alpha \in \Delta\right\}\right)=\vee\left\{\mathscr{C} \lambda_{\alpha}: \alpha \in \Delta\right\} .
$$

Lemma 3. [8] Let $\mathscr{C}:[0,1] \rightarrow[0,1]$ be a complement function that satisfies the monotonic and involutive conditions. Then for any family $\left\{\lambda_{\alpha}: \alpha \in \Delta\right\}$ of fuzzy subsets of $X$,

(i) $\mathscr{C}\left(\sup \left\{\lambda_{\alpha}(x): \alpha \in \Delta\right\}\right)=\inf \left\{\mathscr{C}\left(\lambda_{\alpha}(x)\right): \alpha \in \Delta\right\}$

$$
=\inf \left\{\left(\mathscr{C} \lambda_{\alpha}(x)\right): \alpha \in \Delta\right\}
$$


(ii) $\mathscr{C}\left(\inf \left\{\lambda_{\alpha}(x): \alpha \in \Delta\right\}\right)=\sup \left\{\mathscr{C}\left(\lambda_{\alpha}(x)\right): \alpha \in \Delta\right\}$

$$
=\sup \left\{\mathscr{C}\left(\lambda_{\alpha}(x)\right): \alpha \in \Delta\right\}
$$

Lemma 4. [8] Let $\mathscr{C}$ be a complement function that satisfies the monotonic and involutive conditions. For any two subsets $\lambda$ and $\mu$ we have

(i) $\lambda \leq c l_{\mathscr{C}} \lambda$

(ii) $\lambda$ is fuzzy $\mathscr{C}$-closed $\Leftrightarrow \operatorname{cl}_{\mathscr{C}} \lambda=\lambda$

(iii) $\operatorname{cl}_{\mathscr{C}}\left(\operatorname{cl}_{\mathscr{C}} \lambda\right)=\operatorname{cl}_{\mathscr{C}} \lambda$

(iv) If $\lambda \leq \mu$ then $\operatorname{cl}_{\mathscr{C}} \lambda \leq \operatorname{cl}_{\mathscr{C}} \mu$

(v) $\operatorname{cl}_{\mathscr{C}}(\lambda \vee \mu)=\operatorname{cl}_{\mathscr{C}} \lambda \vee \operatorname{cl}_{\mathscr{C}} \mu$

(vi) $\operatorname{cl}_{\mathscr{C}}(\lambda \wedge \mu)=\operatorname{cl}_{\mathscr{C}} \lambda \wedge \operatorname{cl}_{\mathscr{C}} \mu$

Definition 5. [12] Let $(X, \tau)$ be a fuzzy topological space and $\mathscr{C}$ be a complement function. Then a fuzzy subset $\lambda$ of $X$ is fuzzy $\mathscr{C}$-open in $(X, \tau)$ if $\mathscr{C} \lambda$ is fuzzy closed in $(X, \tau)$.

\section{Fuzzy $\mathscr{C}$-Interior}

In this chapter we define fuzzy $\mathscr{C}$-interior and investigate its properties using arbitrary complement function $\mathscr{C}$.

Definition 6. Let $(X, \tau)$ be a fuzzy topological space. Let $\mathscr{C}$ be the arbitrary complement function. Then for a fuzzy set $\lambda$ of $X$, the fuzzy $\mathscr{C}-$ interior of $\lambda$ is defined as the union of all fuzzy $\mathscr{C}$-open sets contained in $\lambda$, i.e., $\operatorname{int}_{\mathscr{C}}(\lambda)=\vee\{\mu: \mu \leq \lambda, \mu$ is fuzzy $\mathscr{C}-$ open $\}$.

Proposition 7. If the complement function $\mathscr{C}$ satisfies the monotonic and involutive conditions then

(i) $\mathscr{C}(c l \lambda)=\operatorname{int}_{\mathscr{C}}(\mathscr{C} \lambda)$

(ii) $\mathscr{C}\left(\right.$ int $\left._{\mathscr{C}} \lambda\right)=\operatorname{cl}(\mathscr{C} \lambda)$

Proof. (i) From the definition of closure in fuzzy topology we have

$$
c l(\lambda)=\wedge\{\mu: \mu \geq \lambda, \mathscr{C} \mu \in \tau\}
$$




$$
\begin{gathered}
\Rightarrow \operatorname{cl}(\lambda)=\inf \{\mu: \mu \geq \lambda, \mathscr{C} \mu \in \tau\}, \\
\Rightarrow \mathscr{C}(\operatorname{cl}(\lambda))(x)=\mathscr{C}(\inf \{\mu(x): \mu(x) \geq \lambda(x), \mathscr{C} \mu \in \tau\}) .
\end{gathered}
$$

By using lemma 3 it follows that

$$
\mathscr{C}(\operatorname{cl}(\lambda))(x)=\sup \{\mathscr{C} \mu(x): \mathscr{C} \mu(x) \leq \mathscr{C} \lambda(x), \mathscr{C} \mu \in \tau\} .
$$

Since $\mathscr{C}$ satisfies the monotonic condition, replacing $\mathscr{C} \mu$ by $\eta$ we get,

$$
\mathscr{C}(c l(\lambda))(x)=\sup \{\eta(x): \eta(x) \leq \mathscr{C} \lambda(x), \eta \in \tau\} .
$$

Using definition $6, \mathscr{C}(\operatorname{cl}(\lambda))(x)=$ int $_{\mathscr{C}}(\mathscr{C} \lambda)(x)$. This proves that $\mathscr{C}(\operatorname{cl}(\lambda))=$ $\operatorname{int}_{\mathscr{C}}(\mathscr{C} \lambda)$.

(ii) By definition 6,

$$
\begin{aligned}
\operatorname{int}_{\mathscr{C}}(\lambda) & =\vee\{\mu: \mu \leq \lambda, \mu \in \tau\} \\
\Rightarrow \operatorname{int}_{\mathscr{C}}(\lambda) & =\sup \{\mu: \mu \leq \lambda, \mu \in \tau\} \\
\Rightarrow \mathscr{C}\left(\operatorname{int}_{\mathscr{C}}(\lambda)\right) & =\mathscr{C}(\sup \{\mu: \mu \leq \lambda, \mu \in \tau\}) .
\end{aligned}
$$

Using lemma 3, it follows that

$$
\Rightarrow \mathscr{C}\left(\operatorname{int}_{\mathscr{C}}(\lambda)\right)(x)=\inf \{\mathscr{C} \mu(x): \mathscr{C} \mu(x) \geq \mathscr{C} \lambda(x), \mu \in \tau\} .
$$

Since $\mathscr{C}$ satisfies the monotonic condition, replace $\mathscr{C} \mu$ by $\eta$ we get

$$
\mathscr{C}\left(\operatorname{int}_{\mathscr{C}}(\lambda)\right)(x)=\inf \{\eta(x): \eta(x) \geq \mathscr{C} \lambda(x), \mathscr{C} \eta \in \tau\} .
$$

This proves $\mathscr{C}\left(\operatorname{int}_{\mathscr{C}}(\lambda)\right)=\operatorname{cl}(\mathscr{C} \lambda)$.

Proposition 8. Let $\mathscr{C}$ be the complement function that satisfies monotonic and involutive conditions. Then $\operatorname{int}_{\mathscr{C}}(\lambda)=\mathscr{C}(\operatorname{cl}(\mathscr{C} \lambda))$.

Proof. From the definition of closure we have

$$
\begin{gathered}
c l(\mathscr{C} \lambda)=\inf \{\mu: \mu \geq \mathscr{C} \lambda, \mathscr{C} \mu \in \tau\} \\
\Rightarrow \mathscr{C}(\operatorname{cl}(\mathscr{C} \lambda))=\mathscr{C}(\inf \{\mu: \mu \geq \mathscr{C} \lambda, \mathscr{C} \mu \in \tau\}) \\
\Rightarrow \mathscr{C}(\operatorname{cl}(\mathscr{C} \lambda))(x)=\sup \{\mathscr{C} \mu(x): \mathscr{C} \mu(x) \leq \lambda(x), \mathscr{C} \mu \in \tau\} .
\end{gathered}
$$

Since $\mathscr{C}$ satisfies monotonic condition by replacing $\mathscr{C} \mu$ by $\eta$ we get,

$$
\mathscr{C}(\operatorname{cl}(\mathscr{C} \lambda))(x)=\sup \{\eta(x): \eta(x) \leq \lambda(x), \eta \in \tau\} .
$$

Hence $\mathscr{C}(\operatorname{cl}(\mathscr{C} \lambda))=\operatorname{int}_{\mathscr{C}}(\lambda)$. 
Theorem 9. Let $\mathscr{C}$ be a complement function that satisfies boundary, monotonic and involutive conditions. For any two fuzzy subsets $\lambda$ and $\mu$ of a fuzzy topological space $X$, we have

(i) $\operatorname{int}_{\mathscr{C}}(1)=1$

(ii) $\operatorname{int}_{\mathscr{C}}(\lambda) \leq \lambda$

(iii) $\lambda$ is fuzzy $\mathscr{C}$-open $\Leftrightarrow \operatorname{int}_{\mathscr{C}}(\lambda)=\lambda$

(iv) $\operatorname{int}_{\mathscr{C}}\left(\operatorname{int}_{\mathscr{C}}(\lambda)\right)=\operatorname{int}_{\mathscr{C}}(\lambda)$

(v) If $\lambda \leq \mu$ then $\operatorname{int}_{\mathscr{C}}(\lambda) \leq \operatorname{int}_{\mathscr{C}}(\mu)$

(vi) $\operatorname{int}_{\mathscr{C}}(\lambda \vee \mu) \geq \operatorname{int}_{\mathscr{C}}(\lambda) \vee \operatorname{int}_{\mathscr{C}}(\mu)$

(vii) $\operatorname{int}_{\mathscr{C}}(\lambda \wedge \mu)=\operatorname{int}_{\mathscr{C}}(\lambda) \wedge \operatorname{int}_{\mathscr{C}}(\mu)$

Proof. (i) By proposition 8, we have int $\mathscr{C}(\lambda)=\mathscr{C}\left(\operatorname{cl}_{\mathscr{C}}(\mathscr{C} \lambda)\right)$.

Then $\mathscr{C}\left(\operatorname{cl}_{\mathscr{C}}(0)\right)=\mathscr{C}(0) \Rightarrow \operatorname{int}_{\mathscr{C}}(1)=1$.

(ii) It is evident from the definition of $\operatorname{int}_{\mathscr{C}}(\lambda)$ is the largest open set contained in $\lambda$.

(iii) Let $\lambda$ be fuzzy $\mathscr{C}$-open. By definition $6, \mathscr{C} \lambda$ is fuzzy closed. Therefore $\operatorname{cl}(\mathscr{C} \lambda)=\mathscr{C} \lambda$. Using proposition $7, \mathscr{C}\left(\operatorname{int}_{\mathscr{C}}(\lambda)\right)=\operatorname{cl}(\mathscr{C} \lambda)=\mathscr{C} \lambda$. Applying involutive condition we see that $\mathscr{C}\left(\mathscr{C}\left(\right.\right.$ int $\left.\left._{\mathscr{C}}(\lambda)\right)\right)=\mathscr{C}(\mathscr{C} \lambda)$ which implies $\operatorname{int}_{\mathscr{C}}(\lambda)=\lambda$.

Conversely, Let int $\mathscr{C}(\lambda)=\lambda$. Then $\mathscr{C}\left(\right.$ int $\left._{\mathscr{C}}(\lambda)\right)=\mathscr{C}(\lambda)$.Using proposition 7, we have $\operatorname{cl}(\mathscr{C} \lambda)=\mathscr{C} \lambda \Rightarrow \mathscr{C} \lambda$ is fuzzy closed. This shows that $\lambda$ is fuzzy $\mathscr{C}$-open.

(iv) Since $\mathscr{C}\left(\operatorname{int}_{\mathscr{C}}(\lambda)\right)=\operatorname{cl}(\mathscr{C} \lambda)=\operatorname{cl}\left(\mathscr{C}\left(\operatorname{int}_{\mathscr{C}} \lambda\right)\right), \mathscr{C}\left(\right.$ int $\left._{\mathscr{C}} \lambda\right)$ is fuzzy closed and hence $i n t_{\mathscr{C}} \lambda$ is fuzzy $\mathscr{C}$-open. By applying (iii) we get int $t_{\mathscr{C}}\left(\right.$ int $\left._{\mathscr{C}} \lambda\right)=$ $\operatorname{int}_{\mathscr{C}}(\lambda)$.

(v) Suppose $\lambda \leq \mu$. Since $\mathscr{C}$ satisfies monotonic conditions $\mathscr{C} \lambda \geq \mathscr{C} \mu \Rightarrow$ $\operatorname{cl}(\mathscr{C} \lambda) \geq \operatorname{cl}(\mathscr{C} \mu) \Rightarrow \mathscr{C}(\operatorname{cl}(\mathscr{C} \lambda)) \leq \mathscr{C}(\operatorname{cl}(\mathscr{C} \mu))$. Using proposition 8, int $_{\mathscr{C}} \lambda \leq$ int $_{\mathscr{C}} \mu$.

(vi) Since $\lambda \leq \lambda \vee \mu$ and $\mu \leq \lambda \vee \mu \Rightarrow \operatorname{int}_{\mathscr{C}}(\lambda) \leq \operatorname{int}_{\mathscr{C}}(\lambda \vee \mu)$ and $\operatorname{int}_{\mathscr{C}}(\mu) \leq$ $\operatorname{int}_{\mathscr{C}}(\lambda \vee \mu) \Rightarrow \operatorname{int}_{\mathscr{C}}(\lambda) \vee \operatorname{int}_{\mathscr{C}}(\mu) \leq \operatorname{int}_{\mathscr{C}}(\lambda \vee \mu)$. 
(vii) Since $\mathscr{C}$ satisfies the involutive condition, $\operatorname{int}_{\mathscr{C}}(\lambda \wedge \mu)=\operatorname{int}_{\mathscr{C}}(\mathscr{C}(\mathscr{C}(\lambda \wedge \mu)))$. Using proposition 7 , int $\operatorname{ta}_{\mathscr{C}}(\lambda \wedge \mu)=$

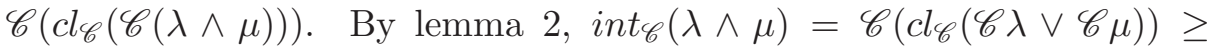
$\mathscr{C}\left(\operatorname{cl}_{\mathscr{C}}(\mathscr{C} \lambda) \vee \operatorname{cl}_{\mathscr{C}}(\mathscr{C} \mu)\right)$. Again by using lemma $2 \operatorname{int}_{\mathscr{C}}(\lambda \wedge \mu)=\mathscr{C}\left(\operatorname{cl}_{\mathscr{C}}(\mathscr{C} \lambda)\right) \wedge$ $\mathscr{C}\left(\operatorname{cl}_{\mathscr{C}}(\mathscr{C} \mu)\right)$. Using proposition $8, \operatorname{int}_{\mathscr{C}}(\lambda \wedge \mu)=\operatorname{int}_{\mathscr{C}}(\lambda) \wedge \operatorname{int}_{\mathscr{C}}(\mu)$. Therefore $\operatorname{int}_{\mathscr{C}}(\lambda \wedge \mu) \geq \operatorname{int}_{\mathscr{C}}(\lambda) \wedge \operatorname{int}_{\mathscr{C}}(\mu)$.

Also $\operatorname{int}_{\mathscr{C}}(\lambda \wedge \mu) \leq \operatorname{int}_{\mathscr{C}}(\lambda)$ and $\operatorname{int}_{\mathscr{C}}(\lambda \wedge \mu) \leq \operatorname{int}_{\mathscr{C}}(\mu) \Rightarrow \operatorname{int}_{\mathscr{C}}(\lambda \wedge \mu) \leq$ $\operatorname{int}_{\mathscr{C}}(\lambda) \wedge \operatorname{int}_{\mathscr{C}}(\mu)$. Then it proves that $\operatorname{int}_{\mathscr{C}}(\lambda \wedge \mu)=\operatorname{int}_{\mathscr{C}}(\lambda) \wedge \operatorname{int}_{\mathscr{C}}(\mu)$.

\section{Fuzzy $\mathscr{C}$-Exterior}

In this chapter we define the fuzzy $\mathscr{C}$-exterior using the arbitrary complement function $\mathscr{C}$ and establish the properties of fuzzy $\mathscr{C}$-exterior.

Definition 10. Let $\lambda$ be a fuzzy subset of a fuzzy topological space $(X, \tau)$ and let $\mathscr{C}$ be a complement function. Then the fuzzy $\mathscr{C}$-exterior of $\lambda$ is defined as $\operatorname{ext}_{\mathscr{C}}(\lambda)=\operatorname{int}_{\mathscr{C}}(\mathscr{C} \lambda)$.

Proposition 11. Let $(X, \tau)$ be a fuzzy topological space and $\mathscr{C}$ be a complement function that satisfies involutive condition. Then for any fuzzy subset $\lambda$ of $X \operatorname{ext}_{\mathscr{C}}(\mathscr{C} \lambda)=\operatorname{int}_{\mathscr{C}}(\lambda)$.

Proof. By using definition 9 , $\operatorname{ext}_{\mathscr{C}}(\mathscr{C} \lambda)=\operatorname{int}_{\mathscr{C}}(\mathscr{C}(\mathscr{C} \lambda))$ and applying involutive condition it proves that $\operatorname{ext}_{\mathscr{C}}(\mathscr{C} \lambda)=\operatorname{int}_{\mathscr{C}}(\lambda)$.

Theorem 12. Let $\mathscr{C}$ be a complement function that satisfies the boundary, monotonic and involutive conditions. For any two subsets $\lambda$ and $\mu$ of a fuzzy topological space, we have

(i) $\operatorname{ext}_{\mathscr{C}}(0)=1$ and $\operatorname{ext}_{\mathscr{C}}(1)=0$

(ii) $\operatorname{ext}_{\mathscr{C}}(\lambda) \leq \mathscr{C} \lambda$

(iii) $\operatorname{ext}_{\mathscr{C}}(\lambda)=\operatorname{ext}_{\mathscr{C}}\left(\mathscr{C}\left(\operatorname{ext}_{\mathscr{C}}(\lambda)\right)\right)$

(iv) $\operatorname{ext}_{\mathscr{C}}(\lambda \vee \mu)=\operatorname{ext}_{\mathscr{C}}(\lambda) \wedge \operatorname{ext}_{\mathscr{C}}(\mu)$

(v) if $\lambda \leq \mu \Rightarrow \operatorname{ext}_{\mathscr{C}}(\mu) \leq \operatorname{ext}_{\mathscr{C}}(\lambda)$

(vi) $\operatorname{int}_{\mathscr{C}}(\lambda) \leq \operatorname{ext}_{\mathscr{C}} \operatorname{ext}_{\mathscr{C}}(\lambda)$ 
Proof. (i) By definition 9, $\operatorname{ext}_{\mathscr{C}}(0)=\operatorname{int}_{\mathscr{C}}(\mathscr{C}(0))=\operatorname{int}_{\mathscr{C}}(1)$ and $\operatorname{ext}_{\mathscr{C}}(1)=$ $\operatorname{int}_{\mathscr{C}}(\mathscr{C}(1))=\operatorname{int}_{\mathscr{C}}(0)$.

(ii) $\operatorname{ext}_{\mathscr{C}}(\lambda)=\operatorname{int}_{\mathscr{C}}(\mathscr{C}(\lambda)) \leq \mathscr{C} \lambda$.

(iii) $\operatorname{ext}_{\mathscr{C}}\left(\mathscr{C}\left(\operatorname{ext}_{\mathscr{C}}(\lambda)\right)\right)=\operatorname{ext}_{\mathscr{C}}\left(\mathscr{C}\left(\operatorname{int}_{\mathscr{C}}(\mathscr{C} \lambda)\right)\right)=\operatorname{int}_{\mathscr{C}}\left(\mathscr{C} \mathscr{C}\left(\operatorname{int}_{\mathscr{C}}(\mathscr{C} \lambda)\right)\right)$ $=\operatorname{int}_{\mathscr{C}}\left(\operatorname{int}_{\mathscr{C}}(\mathscr{C} \lambda)\right)=\operatorname{int}_{\mathscr{C}}(\mathscr{C} \lambda)=\operatorname{ext}_{\mathscr{C}}(\lambda)$.

(iv) $\operatorname{ext}_{\mathscr{C}}(\lambda \vee \mu)=\operatorname{int}_{\mathscr{C}}(\mathscr{C}(\lambda \vee \mu))=\operatorname{int}_{\mathscr{C}}(\mathscr{C} \lambda \vee \mathscr{C} \mu)=\operatorname{int}_{\mathscr{C}}(\mathscr{C} \lambda) \wedge \operatorname{int}_{\mathscr{C}}(\mathscr{C} \mu)=$ $\operatorname{ext}_{\mathscr{C}}(\lambda) \wedge \operatorname{ext}_{\mathscr{C}}(\mu)$.

(v) If $\lambda \leq \mu \Rightarrow \mathscr{C} \mu \leq \mathscr{C} \lambda \Rightarrow \operatorname{int}_{\mathscr{C}}(\mathscr{C} \mu) \leq \operatorname{int}_{\mathscr{C}}(\mathscr{C} \lambda) \Rightarrow \operatorname{ext}_{\mathscr{C}}(\mu) \leq \operatorname{ext}_{\mathscr{C}}(\lambda)$.

(vi) By using (ii), $\operatorname{ext}_{\mathscr{C}}(\lambda) \leq \mathscr{C} \lambda$. Then $\operatorname{ext}_{\mathscr{C}}(\mathscr{C} \lambda) \in \operatorname{ext}_{\mathscr{C}}\left(\operatorname{ext}_{\mathscr{C}}(\lambda)\right)$. But $\operatorname{int}_{\mathscr{C}}(\lambda)=\operatorname{ext}_{\mathscr{C}}(\mathscr{C} \lambda)$. Hence $\operatorname{int}_{\mathscr{C}}(\lambda) \leq \operatorname{ext}_{\mathscr{C}} \operatorname{ext} \mathscr{C}_{\mathscr{C}}(\lambda)$.

\section{Some New Fuzzy Topologies}

In this chapter we characterize the fuzzy topological space using Fuzzy $\mathscr{C}$ Closure, Fuzzy $\mathscr{C}$-Interior and Fuzzy $\mathscr{C}$-Exterior operators.

Theorem 13. Let $(X, \tau)$ be a fuzzy topological space. Let $\mathscr{C}$ be a complement function which satisfies boundary, monotonic and involutive conditions. Let $c_{\mathscr{C}}: I^{X} \rightarrow I^{X}$ be a fuzzy $\mathscr{C}$-closure operator on $X$ such that

1. $\operatorname{cl}_{\mathscr{C}}(0)=0$

2. $\lambda \leq \operatorname{cl}_{\mathscr{C}}(\lambda)$ for each $\lambda$

3. $\operatorname{cl}_{\mathscr{C}}\left(\operatorname{cl}_{\mathscr{C}} \lambda\right)=\operatorname{cl}_{\mathscr{C}}(\lambda)$ for each $\lambda$

4. $\operatorname{cl}_{\mathscr{C}}(\lambda \vee \mu)=\operatorname{cl}_{\mathscr{C}} \lambda \vee \operatorname{cl} \mathscr{C} \mu$ for each $\lambda$ and $\mu$

Then the family $\delta=\left\{\mathscr{C}\left(\operatorname{cl}_{\mathscr{C}} \lambda\right): \lambda \in I^{X}\right\}$ is a fuzzy topology with $\operatorname{cl}_{\delta}(\lambda)=$ $\operatorname{cl}_{\mathscr{C}}(\lambda)$.

Theorem 14. Let $(X, \tau)$ be a fuzzy topological space. Let $\mathscr{C}$ be a complement function which satisfies boundary, monotonic and involutive conditions. Let int ${ }_{\mathscr{C}}: I^{X} \rightarrow I^{X}$ be a fuzzy $\mathscr{C}$-interior operator on $X$ such that

1. $\operatorname{int}_{\mathscr{C}}(1)=1$ 
2. $\operatorname{int}_{\mathscr{C}}(\lambda) \leq \lambda$ for each $\lambda$

3. $\operatorname{int}_{\mathscr{C}}\left(\operatorname{int}_{\mathscr{C}}(\lambda)\right)=\operatorname{int}_{\mathscr{C}}(\lambda)$ for each $\lambda$

4. $\operatorname{int}_{\mathscr{C}}(\lambda \wedge \mu)=\operatorname{int}_{\mathscr{C}}(\lambda) \wedge \operatorname{int}_{\mathscr{C}}(\mu)$ for each $\lambda$ and $\mu$

Then the family $\delta=\left\{\right.$ int $\left._{\mathscr{C}} \lambda: \lambda \in I^{X}\right\}$ is a fuzzy topology with $\operatorname{int}_{\delta}(\lambda)=$ $\operatorname{int}_{\mathscr{C}}(\lambda)$.

Theorem 15. Let $(X, \tau)$ be a fuzzy topological space. Let $\mathscr{C}$ be a complement function which satisfies boundary, monotonic and involutive conditions. Let exte $: I^{X} \rightarrow I^{X}$ be a fuzzy $\mathscr{C}$-exterior operator on $X$ such that

1. $\operatorname{ext}_{\mathscr{C}}(0)=1$ and $\operatorname{ext}_{\mathscr{C}}(1)=0$

2. $\operatorname{ext}_{\mathscr{C}}(\lambda) \leq \mathscr{C} \lambda$

3. $\operatorname{ext}_{\mathscr{C}}(\lambda)=\operatorname{ext}_{\mathscr{C}}\left(\mathscr{C}\left(\operatorname{ext}_{\mathscr{C}}(\lambda)\right)\right)$

4. $\operatorname{ext}_{\mathscr{C}}(\lambda \vee \mu)=\operatorname{ext}_{\mathscr{C}}(\lambda) \wedge \operatorname{ext}_{\mathscr{C}}(\mu)$

Then the family $\delta=\left\{\operatorname{ext}_{\mathscr{C}} \lambda: \lambda \in I^{X}\right\}$ is a fuzzy topology with $\operatorname{ext}_{\delta}(\lambda)=$ $\operatorname{ext}_{\mathscr{C}}(\lambda)$.

\section{References}

[1] L.A. Zadeh, Fuzzy sets, Information and Control, 8 (1965), 338-353, doi: 10.1016/S00199958(65)90241-X.

[2] C.L.Chang, Fuzzy topological spaces, J. Math. Anal. Appl., 24 (1968), 182-190, doi: 10.1016/0022-247X(68)90057-7.

[3] Rosenfeld, Fuzzy digital topology, Information and Control, 40 (1979), 76-87, doi: 10.1016/S0019-9958(79)90353-X.

[4] A.S. Mashhour, M.H. Ghanim, Fuzzy closure space, J. Math. Anal. Appl., 106 (1985), 154-170, doi: 10.1016/0022-247X(85)90138-6.

[5] George J. Klir, Bo Yuan, Fuzzy Sets and Fuzzy Logic Theory and Applications, Prentice Hall,Inc., 2005.

[6] Wenzhong Shi, Kimfung Liu, A fuzzy topology for computing the interior, boundary and exterior of spatial objects quantitatively in GIS, Computers and Geosciences, 33 (2007), 898-915, doi: 10.1016/j.cageo.2006.10.013.

[7] Kimfung Liu, Wenzhong Shi, Quantitative fuzzy topological relations of spatial objects by induced fuzzy topology, International Journal of Applied Earth Observation and Geoinformation, 11 (2009), 38-45, doi: 10.1016/j.jag.2008.06.001.

[8] K. Bageerathi, G. Sutha, P. Thangavelu, A generalization of fuzzy closed sets, International Journal of Fuzzy Systems and Rough Systems, 4, No. 1 (2011), 1-5. 
[9] Guo Jifa, Cui Tiejun, Discussion on Type-1 fuzzy boundary and research on boundary definition of high order fuzzy region, TELKOMNIKA Indonesian Journal of Electrical Engineering, 10, No. 6 (2012), 1207-1213, doi: 10.11591/telkomnika.v10i6.1544.

[10] Homa Fashandi, J.F. Peters, Crisp and fuzzy topological interior and closure operators with inclusion degree theory and applications, Fundamenta Informaticae, 122 (2013), 207-225.

[11] H.C. Chamuah, B.C. Chetia, Application of fuzzy topological relation in flood prediction, International Journal of Computer Applications, 122, No. 7 (2015), 8-13, doi: org/10.5120/21710-4830.

[12] P. Xavier, P.Thangavelu, Generalization of fuzzy open sets and fuzzy closed sets via complement functions, In: Proceeding of International Conference on Electrical, Electronics, and Optimization Techniques (ICEEOT) (2016), 889-891. 
